\title{
Autonomous Detection of the Loss of a Wing for Underwater Gliders
}

\author{
Enrico Anderlini \\ Catherine A. Harris \\ Georgios Salavasidis \\ Department of Mechanical Engineering Marine Autonomous and Robotic Systems Marine Autonomous and Robotic Systems \\ University College London \\ National Oceanography Centre \\ Southampton, UK \\ National Oceanography Centre \\ London, UK \\ E.Anderlini@ucl.ac.uk \\ Southampton, UK \\ Alvaro Lorenzo \\ Alexander B. Phillips \\ Giles Thomas \\ Marine Autonomous and Robotic Systems Marine Autonomous and Robotic Systems Department of Mechanical Engineering \\ National Oceanography Centre \\ National Oceanography Centre \\ Southampton, UK \\ University College London \\ Southampton, UK \\ London, UK
}

\begin{abstract}
Over the past five years, two of the Slocum underwater gliders operated by the UK National Oceanography Centre have lost a wing mid-mission without the pilot being aware of the problem until the point of vehicle retrieval. In this study, the steady-state data collected by gliders during the two deployments has been analysed to develop a fault detection system. From the data analysis, it is clear that the loss of the wing was a sudden event for both gliders. The main changes to the system dynamics associated with the event are an increase in the positive buoyancy of the glider and the occurrence of a roll angle on the side of the lost wing, with significant difference between dives and climbs. Hence, a simple effective system for the detection of the wing loss has been designed using the roll angle. Since sensors are known to fail and the roll sensor is non-critical to the operation of the glider, a back-up diagnostics system has been developed based on the dynamic model of the vehicle, capturing the change in buoyancy. Both systems are able to correctly detect the loss of the wing and notify pilots, who can re-plan missions to safely recover the vehicle.
\end{abstract}

Index Terms-underwater glider, fault detection, fault isolation, system identification, wing loss

\section{INTRODUCTION}

Underwater gliders (UGs) are a type of autonomous underwater vehicle that are extensively used for the exploration and study of the oceans [1]. The vehicles achieve vertical motion in the water by changing their buoyancy through a variable buoyancy device (VBD). Wings generate a forward motion component from the vertical motion. Hence, UGs travel in a characteristic sawtooth pattern in the vertical plane. Their simple propulsion system, which consists of the VBD, pitch control and either roll control or a rudder, is highly efficient. Therefore, although they operate at low velocities $(\approx 0.3 \mathrm{~m} / \mathrm{s})$, a single UG deployment can last for several months.

The National Oceanography Centre (NOC) in the UK is developing a new command-and-control system for efficient marine autonomous systems fleet management as part of the Oceanids project funded by the Industrial Strategy Challenge

C. Harris, G. Salavasidis, A. Lorenzo Lopez and A. B. Phillips contributions were funded under the NERC/ISCF Oceanids programme.
Fund [2], [3]. The aim of the system is to facilitate the overthe-horizon operation of the ever-increasing fleet of AUVs in the UK's National Marine Equipment Pool, which includes over 30 UGs. As part of an on-going collaboration, the authors have previously prototyped a recommender system to help pilots trim and set-up Seaglider UGs [4], [5]. In this study, the aim is to address a different operational challenge: identifying when a glider loses a wing. Wings and their locking mechanisms are not as able as the pressure hull to withstand large impact loads. Hence, in case of significant collisions, wings can detach from the UG body.

The reliability of UGs is analysed thoroughly in [6], with the authors collecting failure data from most European operators. However, the loss of a wing was not reported as a problem. Therefore, to our knowledge, this is the first study that investigates the response of an UG after the loss of one wing mid-mission. Over the past five years, two of the Slocum UGs operated by NOC have lost one wing during a deployment without the pilot noticing the event until retrieval. In this article, the data from those deployments is analysed to investigate the impact of the loss of wing on the dynamics of the vehicle. A simple but effective rule- and model-based, remote, real-time fault detection method is then developed.

\section{Slocum Gliders DATA}

\section{A. Slocum Underwater Gliders}

Slocum is a type of UGs manufactured by Teledyne Webb Research that have been in operation since the late nineties [7], [8]. The Slocum G2 [9], the second version of the UG, is considered in this study to match the type of vehicles that lost a wing. In particular, the analysed UGs are rated for a maximum depth of $200 \mathrm{~m}$. As shown in Fig. 1, the Slocums are actuated by a VBD, which consists in an oil bladder that can be extended or retracted from the pressure hull. When the bladder is outside the pressure hull, the vehicle's displacement increases and so does its net buoyancy and vice versa. The vehicles considered in this study are limited to changes in the 
VBD volume of $\pm 250 \mathrm{~cm}^{3}$. Furthermore, pitch is controlled by shifting the position of one movable battery pack with a dedicated mechanism. Yaw is controlled through a rudder, which is magnetically coupled to a servo motor to avoid an opening in the pressure hull.

Example time series data for a typical dive cycle can be seen in Fig. 2, $z$ indicates the vertical position of the vehicle in the water column (positive upwards), which is measured by an on-board pressure sensor. Its time-derivative yields $\dot{z}$, the vertical velocity. The actuators' control signals are the volume of the VBD, $V_{\mathrm{vbd}}$, the position of the moving battery pack, $x_{\mathrm{b}}$, and the rudder angle, $\delta_{\mathrm{r}}$. The roll, $\phi$, and pitch, $\theta$, angles are measured by tilt sensors, while a compass indicates the heading angle, $\psi$. In this article, the difference of the instantaneous yaw angle and the mean yaw angle over the whole dive cycle, $\bar{\psi}$, is used instead to favour the body-fixed over the inertial reference frame.

From Fig. 1 and Fig. 2a, it is clear that Slocums can perform multiple "yos" per dive (two in this particular case). This means that the vehicle can sample the water column multiple times before returning to the surface to send and receive data by satellite and get a new position fix [9]. The data sent ashore needs to be decimated to reduce the time that the vehicle spends on the surface and thus the risks of surface collisions and wind-induced drift, the power expenditure and the financial cost associated with the transmission of the data by satellite (specifically, via Iridium). This data usually includes the vehicle's orientation, its depth (from which the vertical velocity can be obtained), the actuator's signals, the capacity and voltage of the battery, the estimated location, samples of the scientific data of interest and warnings from on-board health monitoring systems. The decimation means that the samples have a low rate, typically with a time step of 30-60 s for signals that are considered of least importance (e.g. the roll angle) and $10 \mathrm{~s}$ for the signals that are of most interest, e.g. the scientific data. Although Fig. 2 a shows that the glider returns to the surface after every yo, the minimum depth can be set to be lower to avoid marine traffic and biofouling [10]. In the analysed dataset, each dive can consist of up to 14 yos and last up to four hours.

Fig. 3 shows an example dive cycle for the same UG (Slocum 436) after the loss of a wing. As compared with Fig. 2, the major differences are that:

- the VBD signal is offset from the vertical velocity signal in Fig. 3 ,

- the roll angle changes between descents and ascents in Fig. 3d,

- heading errors are larger and periodic, resulting in larger corrective rudder angles in Fig. 3 .

To control UGs, pilots rely on the surface dialogue, which is a summary of the vehicle's health status, current mission plan, last GPS position and the decimated data from past dives. Typically, during normal operation, the remote pilots will first check the surface dialogue for errors, warning and oddities from the glider subsystems, along with the dive profile to ensure it is symmetrical and the glider is reaching the target depth. The most common errors identified on board are relatively mild aborts, e.g. glider stalls, behaviour errors, and communication interruptions [8]. Progress towards the target waypoint is also considered, along with a check of the battery health and consumption. This full check is usually performed once per day, with the pilot making smaller observations more regularly after each dive. Therefore, pilots are only likely to look into the flight parameters in detail if the glider is reporting errors, is failing to dive correctly or is not making progress towards a waypoint. Hence, issues with roll, for instance, can go unnoticed.

\section{B. Dataset Description}

This study involves the data measured by Slocum G2 gliders over two deployments operated by the NOC during which each vehicle lost one wing $[11]$. A summary of the missions, including UG ID, mission date and location, can be found in Table I]

TABLE I

SUMMARY OF THE ANALYSED DEPLOYMENTS [11].

\begin{tabular}{ccccc} 
No. & Glider ID & Date & Location & Duration [days] \\
\hline 1 & 304 & 2019 & North Sea & 76.9 \\
2 & 436 & 2019 & North Sea & 89.8
\end{tabular}

The missions were part of project ALTERECO: "An Alternative Framework to Assess Marine Ecosystem in Shelf Seas" (NE/P013902/2). In this project, the UGs were used to validate a novel monitoring framework to deliver improved spatial and temporal understanding of the health and function of shelf sea ecosystem [12].

Gliders were deployed and recovered from a mixture of large and small research vessels as well as fishing boats. Once deployed, gliders undertook sustained observations for multimonth periods, undertaking repeated transects in the relevant operating area. Once the vehicles were deployed, the only human intervention was via remote pilots, up until the point of recovery. By cycling the gliders, sustained observations can be extended to multiple years.

\section{WING LOSS DETECTION}

Two separate methods are developed for the detection of the loss of wing: a rule-based and a model-based solution. Rule-based diagnostics are an approach that relies on bespoke heuristics, usually in the form of if-then statements, obtained from designers' observations of the system [13]. Conversely, model-based diagnostics use a model constructed from indepth knowledge of the system dynamics.

\section{A. Data Cleaning}

As UGs operate in steady-state conditions for the most of the flight, the dataset is cleaned to retain only steady-state conditions. Firstly, the recovery-mode data is converted from binary to ASCII format using the Python dbdreader module ${ }^{2}$

\footnotetext{
${ }^{1}$ The data is openly available on request from BODC at enquiries@bodc.ac.uk

${ }^{2}$ https://pypi.org/project/dbdreader/
} 


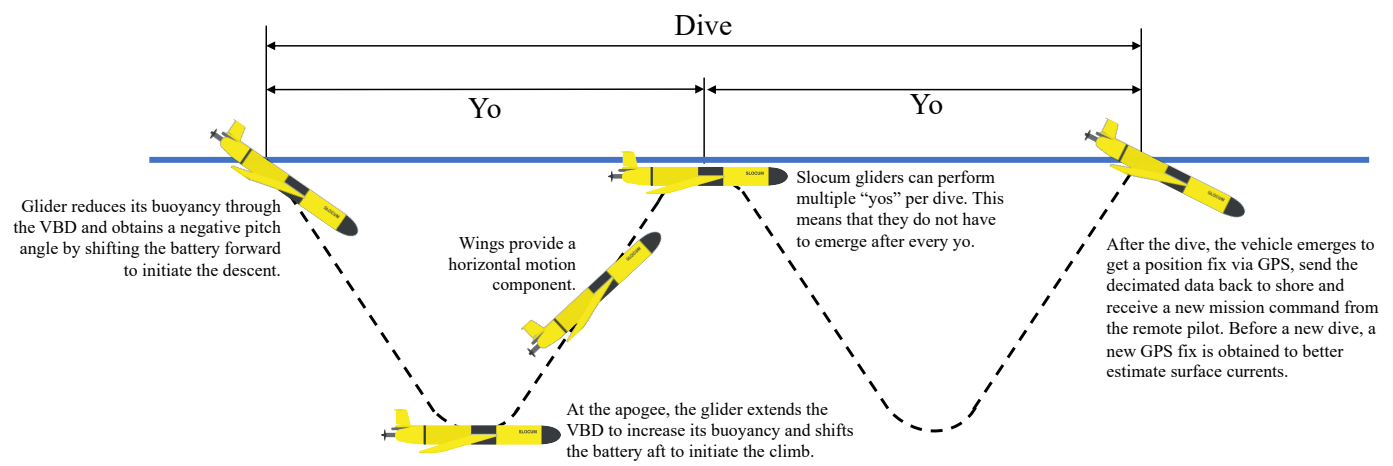

Fig. 1. Diagram showing the concept of operation of a Slocum UG. The drawing is not to scale: the analysed vehicles reach their apogee at 200-m-depth and and have glide path angles with a magnitude in the range $\left(15^{\circ}, 30^{\circ}\right)$.

a)

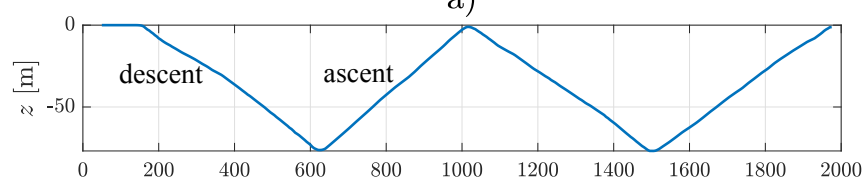

b)

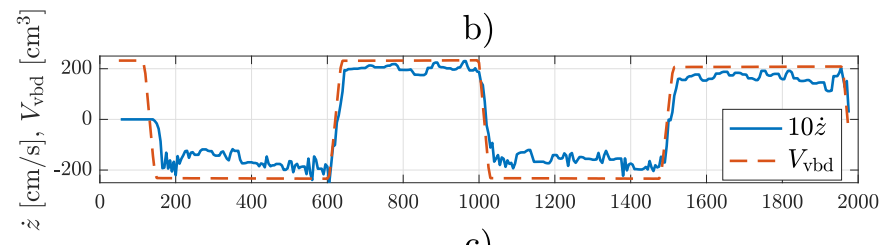

c)

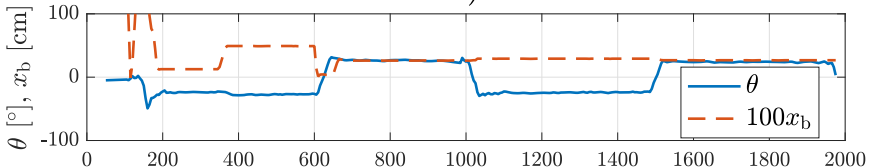

d)

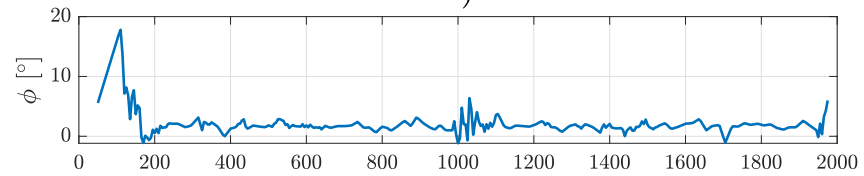

e)

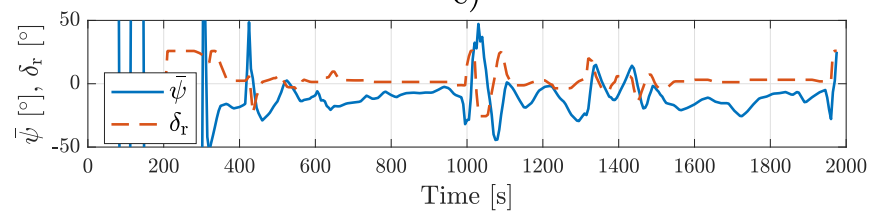

Fig. 2. Example dive cycle of an intact Slocum glider.

Subsequently, the points are imported into the MATLAB environment. Then, all dive cycles for which any of the signals of interest are unavailable are removed. Additionally, a time vector starting from $0 \mathrm{~s}$ is created for every cycle. In a further cleaning sweep, all cycles with either a maximum depth $<25 \mathrm{~m}$ or less than 10 time stamps are removed. The signals from the separate navigation and scientific CPUs are synced through the pressure signal, which is measured by both units. All signals are resampled for exactly the same time stamps, with a time step of $5 \mathrm{~s}$.
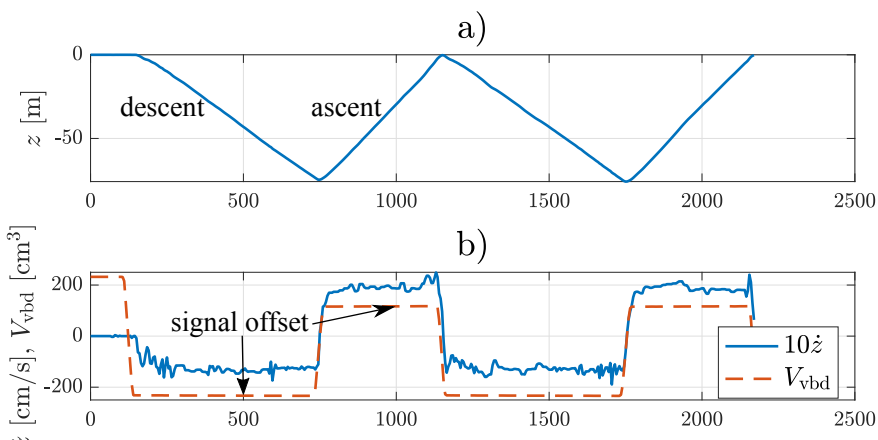

c)

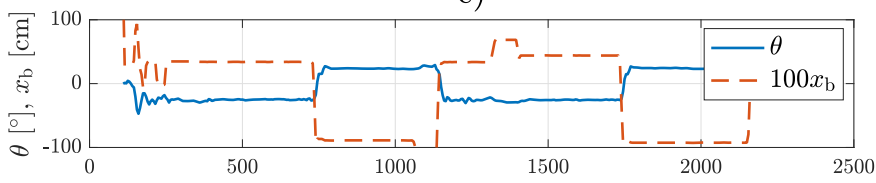

d)

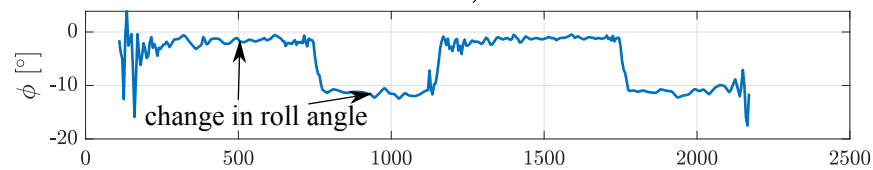

e)

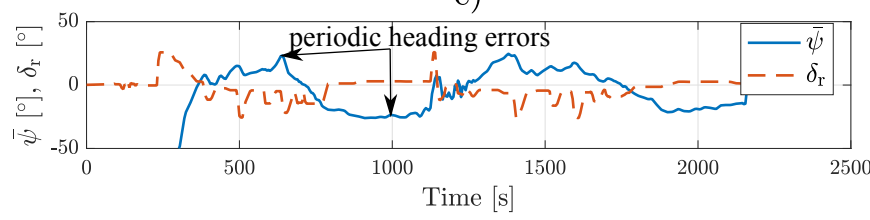

Fig. 3. Example dive cycle of a Slocum glider after the loss of one wing.

Afterwards, the variables of interest, such as vertical velocity and water density, are computed from the raw signals. Only steady-state data are kept by removing points which present significant changes in the actuators values, high vertical acceleration or high rotational velocities. All points which present a nonnumeric value for any signals are removed. The data are then merged once again for each cycle. Any empty cells at this stage are cleared. 


\section{B. Rule-Based Diagnostics}

From the analysis of Fig. 2 and Fig. 3, the clearest indicator of the loss of a wing is recognised as the difference in the value of the roll angle between dives and climbs, as described in Sec. III Since the roll angle is stationary over each descent and ascent even for the damaged glider, a simple but effective initial tool for the detection of the wing loss can be created by averaging the roll angle over the descent and ascent of all yos in each cycle. Hence, the difference of the mean steady-state roll angle in ascents and descents in a single dive can be used as an indicator of wing loss:

$$
\Delta \operatorname{mean}(\phi)=\overline{\phi_{\mathrm{a}}}-\overline{\phi_{\mathrm{d}}}
$$

where $\overline{\phi_{\mathrm{a}}}$ and $\overline{\phi_{\mathrm{d}}}$ indicate the mean roll angle in ascents and descents, respectively. The threshold value for the indicator will be identified in Sec. IV

This method is highly effective and computationally inexpensive. However, as the data relies on a single sensor, the tilt sensor for roll, the scheme is susceptible to sensor calibration and malfunction. For instance, the failure of the pitch tilt sensor was observed in [14] for a Seaglider UG. Therefore, alternative back-up methods that rely on different sensors are required.

\section{Model-Based Diagnostics}

The free-body diagram of the equilibrium condition for the steady-state flight is shown graphically in Fig. 4a and Fig. 4p for descents and ascents, respectively. $B$ indicates the net buoyancy, $L$ the lift and $D$ the drag force. $U$ is the surge velocity component in the body-fixed frame, $\theta$ the pitch, $\alpha$ the attack and $\beta$ the glide-path angles.

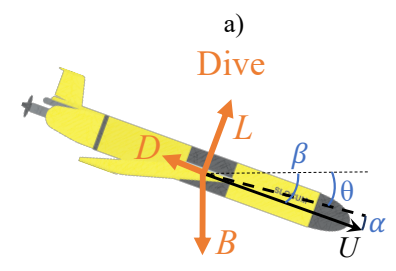

b)

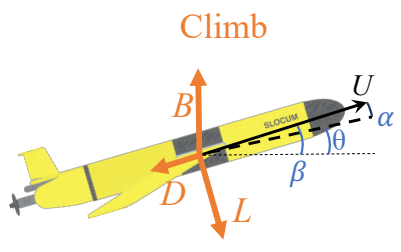

Fig. 4. Free-body diagram of the intact glider in descents (a) and ascents (b) (profile view).

In both descents and ascents, the force balance yields [15]

$$
B-L \cos \beta-D \sin \beta=0,
$$

where $\beta=\theta+\alpha$. According to the standard dynamic model for Slocum UGs in steady-state conditions [15], the drag and lift forces can be modelled as

$$
L=\frac{1}{2} k_{\mathrm{L}} \alpha \rho S U^{2}
$$
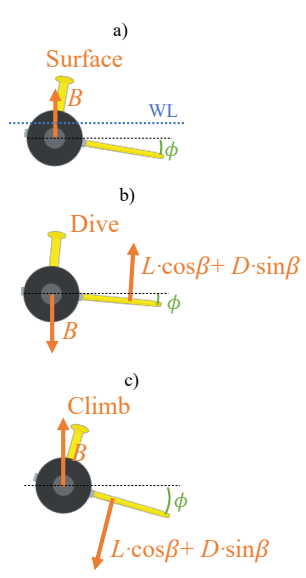

Fig. 5. Free-body diagram of the glider after the loss of the starboard wing on the surface (a), in descents (b) and ascents (c) (body plan view).

$$
D=\frac{1}{2}\left(k_{\mathrm{D}, 0}+k_{\mathrm{D}, \mathrm{L}} \alpha^{2}\right) \rho S U^{2},
$$

where $\rho$ is the water density, $S$ is the wetted surface area, $k_{\mathrm{L}}, k_{\mathrm{D}, 0}$ and $k_{\mathrm{D}}$ are constants used to compute the lift and drag coefficients. The water density is obtained from the water pressure, salinity and temperature using the Gibbs Seawater Toolbox [16]. These properties are measured by a CTD sensor, which, as part of the sensor bay, relies on a separate CPU from the navigation unit.

The net buoyancy force can be computed as

$B=g\left\{-m+\rho\left[V_{0}\left(1-\epsilon_{c} p+\alpha_{T}\left(T-T_{0}\right)\right)+V_{\mathrm{vbd}}\right]\right\}+\delta B$,

where $g$ is the gravitational acceleration, $m$ is the UG mass, $V_{0}$ its reference volume, $\epsilon_{c}$ the absolute compressibility of the pressure hull and $\alpha_{T}$ its thermal expansivity, with the reference temperature $T_{0} . p$ is the water pressure and $T$ its temperature. The additional term $\delta B$ represents a possible offset in buoyancy, which is necessary to account for changes to the vehicle's mass.

The loss of the wing will cause the glider to become more buoyant, since the composite wing is denser than water [9]. Additionally, a decrease in the skin friction drag will counteract an increase in induced drag. Conversely, the lift force that can be generated will significantly decrease. As a result, the controller will need to adapt to an offset in the VBD change in descents and ascents to account for the change in the vehicle net buoyancy. Furthermore, the centres of gravity and buoyancy and the point at which the lift and drag act to shift longitudinally and sideways. The pitching moment is counteracted by a change in the battery position, while the yawing moment is corrected by the rudder. Conversely, the shift in the position of the centres of buoyancy, gravity, lift and drag will cause a roll moment that at equilibrium results in a list angle, as summarised in Fig. 5. Hence, (2) is now updated to

$$
B-\sin \phi(L \cos \beta+D \sin \beta)=0 .
$$


Ideally, the fault detection system should be able to identify problems other than purely the loss of wings. Hence, to ensure generality, the system will be based on the established dynamic model for Slocum UGs to predict the steady-state vertical velocity considering only motions in surge, heave and pitch. Substituting (3), (4) and (5) into (2), it is possible to express the vertical velocity predicted by the model, $\dot{z}_{\mathrm{m}}$, as follows [15]:

$$
\begin{array}{r}
\dot{z}_{\mathrm{m}}=\sqrt{U^{2}} \sin \beta, \text { where } \\
U^{2}=\frac{B \sin \beta}{0.5 \rho S\left(k_{\mathrm{D}, 0}+k_{\mathrm{D}, \mathrm{L}} \alpha^{2}\right)}, \\
\alpha=\frac{k_{\mathrm{D}, 0}+k_{\mathrm{D}, \mathrm{L}} \alpha^{2}}{k_{\mathrm{L}} \tan (\beta)} .
\end{array}
$$

Equation (7c) requires an iterative solution. Additionally, an optimisation needs to be run to find the desired parameters. The cost function is expressed as:

$$
J=\left[\frac{1}{I} \sum_{i=1}^{I}\left(\dot{z}_{i}-\dot{z}_{\mathrm{m}, i}\right)^{2} \forall i \in \mathbb{R}\right]+\left[\sum_{j=1}^{J} j \forall j \notin \mathbb{R}\right] .
$$

In (8), $i$ indicate all points for which a numeric cost value is obtained, while $j$ all points for which $U^{2}<0$. Imposing an additional cost on the number of nonnumeric values speeds up convergence as compared with Merckelbach, et al. [15].

The values for some parameters are known; namely, $g=$ $9.81 \mathrm{~m} / \mathrm{s}^{2}, \epsilon_{c}=6.4 \times 10^{-6} \mathrm{dbar}^{-1}$ and $\alpha_{T}=5.3 \times 10^{-5}{ }^{\circ} \mathrm{C}^{-1}$ for Slocum G2 UGs (from the manufacturer), $S=0.1 \mathrm{~m}^{2}[15]$, and $m, V_{0}$ and $T_{0}$ are measured during the ballasting tests in a tank before each deployment. The values for the analysed UGs are shown in Table II Therefore, the optimisation is run to find the values of the parameters $k_{\mathrm{L}}, k_{\mathrm{D}, 0}, k_{\mathrm{D}, \mathrm{L}}$ and $\delta B$.

TABLE II

SUMMARY OF THE HYDROSTATICS FOR THE ANALYSED SLOCUM UGS.

\begin{tabular}{cccc} 
Glider ID & $m[\mathrm{~kg}]$ & $V_{0}\left[\mathrm{~cm}^{3}\right]$ & $T_{0}\left[{ }^{\circ} \mathrm{C}\right]$ \\
\hline 304 & 59.044 & 57615.8 & 19.23 \\
436 & 65.281 & 63716.5 & 19.45
\end{tabular}

From a fault detection perspective, it is interesting to track changes over each individual dive cycle. Although it is possible to solve for $k_{\mathrm{L}}, k_{\mathrm{D}, 0}, k_{\mathrm{D}, \mathrm{L}}$ and $\delta B$ simultaneously [15], the range of vertical velocity and pitch angle values in individual dive cycles (even if consisting of multiple yos) is unlikely to be comprehensive. A much larger dataset (usually over the whole deployment) is typically needed to obtain all parameters [15]. Hence, the values of the lift and induced drag coefficients are preset to values found in [15]: $k_{\mathrm{L}}=7.5 \mathrm{rad}^{-1}$ and $k_{\mathrm{D}, \mathrm{L}}=3 \mathrm{rad}^{-2}$ for Slocum G2 UGs. Then, a global search optimisation is run to find $k_{D, 0}$ and $\delta B$ for each dive cycle. A scatter search [17] is used to generate trial points within the search space from which a constrained, nonlinear programming solution is found based on a trust region method based on interior point techniques [18]. The scatter search algorithm then assesses the cost function of the solutions to update the trial points and continue the minimisation until convergence onto the global optimum. The MATLAB GlobalSearch and fmincon tools were used in the practical implementation, with default settings. The drag coefficient and buoyancy offset values were constrained to $k_{D, 0} \in[0,0.4]$ and $\delta B \in[-3,3] \mathrm{N}$, respectively.

\section{RESUlTS AND Discussion}

\section{A. Wing Loss Detection}

Figure 6 shows the time variation of the difference of the mean roll angle during the steady-state portion of ascents and descents for the two affected deployments, Slocum 304 and Slocum 436. In Fig. 7, the time variation of the lift coefficient and buoyancy offset can be seen. From Fig. 6 7 , it is clear that wing loss was a sudden event for both gliders, with an impact of the UG with marine traffic being the likely cause. Both the change in the mean roll angles and the buoyancy offset are clear indicators of the onset of wing loss, whilst the drag coefficient is unaffected. Furthermore, from Fig. 6, it is possible to deduce that Slocum 304 has lost the right wing, while Slocum 436 the left wing.

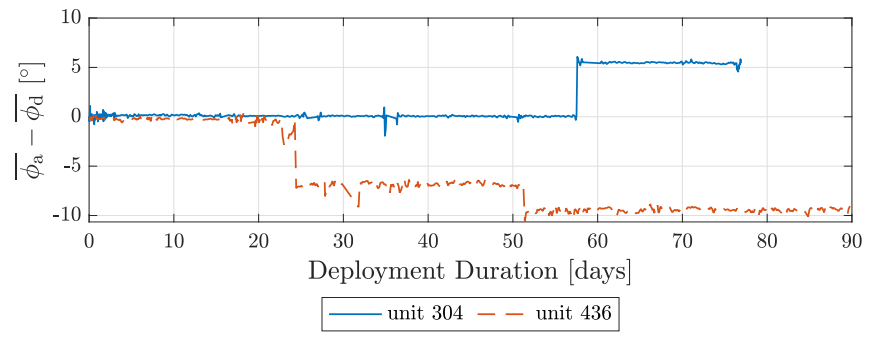

Fig. 6. Time variation of the difference of the mean roll angle during the steady-state portion of ascents and descents.

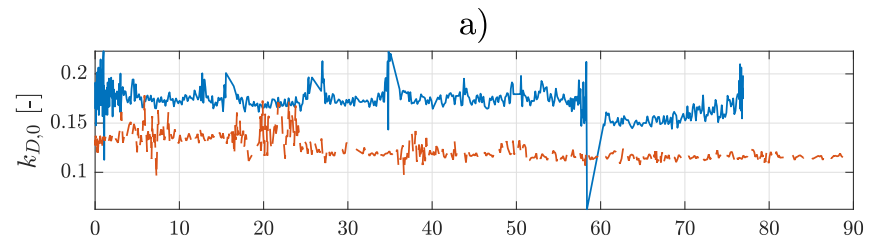

b)

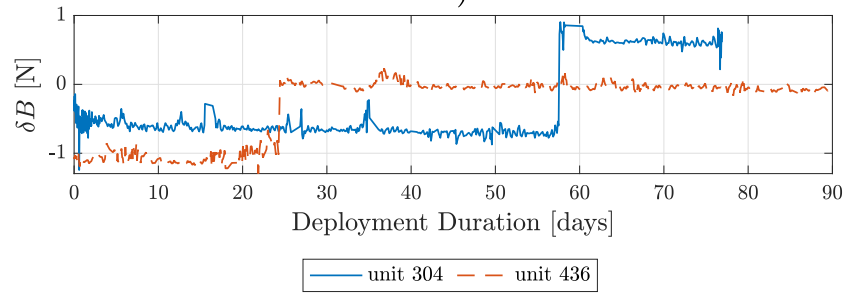

Fig. 7. Time variation of the lift coefficient and buoyancy offset.

Whilst the rule-based fault detection method is simplest and most efficient, the model-based solution is as accurate and relies only on sensors (the depth, pitch, VBD and battery position sensors) that are fundamental for the operation of 
the UG. Hence, the model-based approach offers a redundant system that may be used to continue operations in case the roll sensor fails.

Both systems have already been used to correctly detect the loss of the wing for an additional deployment during April 2020 by interfacing with the NOC's fleet management system.

\section{B. Cost of Transport}

After identifying the two portions of the dataset where the vehicle operated with and without wing, the energetic cost of transport (COT), quantified as the ratio of the energy expenditure and the product of the vehicle's mass and distance travelled [19], is computed to assess the impact of the loss of one wing on the UG's dynamics.

Table III displays the mean and standard deviation of the COT and horizontal velocity for the clean and biofouled UG in dives with a maximum depth of $200 \mathrm{~m}$. The distance travelled during the dive is computed from the latitude and longitude at the start and end of the dive, when the UG receives a GPS fix. Similarly, the dive duration is computed from the difference of the respective time stamps.

TABLE III

MEAN AND STANDARD DEVIATION OF THE COT AND HORIZONTAL VELOCITY FOR A 200-M DIVE.

\begin{tabular}{lc|cc|cc} 
& & \multicolumn{2}{c}{ Slocum 304 } & \multicolumn{2}{c}{ Slocum 436 } \\
Variable & Units & Intact & Damaged & Intact & Damaged \\
\hline$\overline{\mathrm{COT}}$ & {$\left[\mathrm{J} \mathrm{m}^{-1} \mathrm{~kg}^{-1}\right]$} & 0.143 & 0.155 & 0.224 & 0.252 \\
$s_{\mathrm{COT}}$ & {$\left[\mathrm{J} \mathrm{m}^{-1} \mathrm{~kg}^{-1}\right]$} & 0.188 & 0.160 & 0.223 & 0.293 \\
$\overline{\dot{x}}$ & {$\left[\mathrm{~m} \mathrm{~s}^{-1}\right]$} & 0.267 & 0.232 & 0.232 & 0.220 \\
$s_{\dot{x}}$ & {$\left[\mathrm{~m} \mathrm{~s}^{-1}\right]$} & 0.114 & 0.101 & 0.109 & 0.121
\end{tabular}

As can be seen in Table III, the wing loss results in an increase in the COT of approximately $10 \%$ for the two UGs, although the different length of the endpoints of each dive cycle, number of yos per dive and the effect of ocean currents causes high variance. Similarly, the mean horizontal speed through the water drops by $5 \%-12 \%$.

\section{CONCLUSIONS}

UGs have been observed to lose one wing during operations. This sudden phenomenon causes a change in the roll angle between descents and ascents, an increase in the net buoyancy, higher variance in the yaw and rudder angles in the glider, a rise in the energetic cost of transport and a drop in the horizontal speed through the water.

A simple rule-based method has been developed that precisely detects wing loss from the roll angle. A model-based system capturing the change in buoyancy offers redundancy and the same level of accuracy, whilst enabling operations even in the event that the roll data is unavailable. The developed tools have already been used to successfully detect wing loss and inform pilots of the event during a new deployment in 2020 , so that a safe retrieval could be planned.

\section{REFERENCES}

[1] D. L. Rudnick, "Ocean Research Enabled by Underwater Gliders," Annual Review of Marine Science, vol. 8, no. 1, pp. 519-541, 2016.

[2] J. Farley, A. W. Morris, O. D. Jones, C. A. Harris, and A. Lorenzo, "Marine Science from an Armchair: A Unified Piloting Framework for Autonomous Marine Vehicles," in IEEE Oceans, Marseille, France, 2019, pp. 1-10.

[3] C. A. Harris, A. Lorenzo-Lopez, O. Jones, J. J. Buck, A. Kokkinaki, S. Loch, T. Gardner, and A. B. Phillips, "Oceanids C2: An Integrated Command, Control, and Data Infrastructure for the Over-the-Horizon Operation of Marine Autonomous Systems," Frontiers in Marine Science, vol. 7, jun 2020.

[4] E. Anderlini, C. Harris, M. Woo, and G. Thomas, "A New Recommender System for Determining Trim and Flight Parameters of Seagliders," in 29th International Symposium on Ocean and Polar Engineering. Honolulu, HI: ISOPE, 2019.

[5] E. Anderlini, C. Harris, A. B. Phillips, A. Lorenzo Lopez, M. Woo, and G. Thomas, "Towards autonomy: A recommender system for the determination of trim and flight parameters for Seagliders," Ocean Engineering, vol. 189, p. 106338, oct 2019.

[6] M. Brito, D. Smeed, and G. Griffiths, "Underwater glider reliability and implications for survey design," Journal of Atmospheric and Oceanic Technology, vol. 31, no. 12, pp. 2858-2870, 2014.

[7] D. C. Webb, P. J. Simonetti, and C. P. Jones, "SLOCUM: An underwater glider propelled by environmental energy," IEEE Journal of Oceanic Engineering, 2001.

[8] O. Schofield, J. Kohut, D. Aragon, L. Creed, J. Graver, C. Haldeman, J. Kerfoot, H. Roarty, C. Jones, D. Webb, and S. Glenn, "Slocum Gliders: Robust and ready," Journal of Field Robotics, vol. 24, no. 6, pp. 474 485, 2007.

[9] Teledyne Webb Research, "Slocum G2 Glider Operators Manual," Teledyne Webb Research, Tech. Rep., 2012. [Online]. Available: www.webbresearch.com

[10] C. D. I. Haldeman, D. K. Aragon, T. Miles, Scott M. Glenn, and A. G. Ramos, "Lessening biofouling on long-duration AUV flights: Behavior modifications and lessons learned," in MTS/IEEE Oceans. Monterey, California, USA: MTS/IEEE, 2016.

[11] BODC, "Glider inventory," 2019. [Online]. Available: https://www.

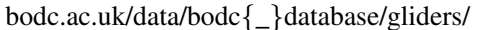

[12] Matthew R. Palmer, C. Williams, A. Akpinar, C. Mahaffey, T. Hull, and M. Toberman, "AlterEco: An Alternative Framework to Assess Marine Ecosystem Functioning in Shelf Seas," 2020. [Online]. Available: https: //meetingorganizer.copernicus.org/EGU2020/EGU2020-18354.html

[13] K. Hamilton, D. M. Lane, K. E. Brown, J. Evans, and N. K. Taylor, "An integrated diagnostic architecture for autonomous underwater vehicles," Journal of Field Robotics, vol. 24, no. 6, pp. 497-526, jun 2007.

[14] E. Frajka-Williams, C. C. Eriksen, P. B. Rhines, and R. R. Harcourt, "Determining vertical water velocities from Seaglider," Journal of Atmospheric and Oceanic Technology, vol. 28, no. 12, pp. 1641-1656, 2011.

[15] L. Merckelbach, A. Berger, G. Krahmann, M. Dengler, and J. R. Carpenter, "A dynamic flight model for Slocum gliders and implications for turbulence microstructure measurements," Journal of Atmospheric and Oceanic Technology, vol. 36, pp. 281-296, jan 2019.

[16] T. McDougall and P. Barker, "Getting started with TEOS-10 and the Gibbs Seawater (GSW) Oceanographic Toolbox," SCOR/IAPSO WG 127, p. $28,2011$.

[17] Z. Ugray, L. Lasdon, J. Plummer, and F. Glover, "Scatter Search and Local NLP Solvers : A Multistart Framework for Global Optimization," Information Systems, vol. 19, no. 3, pp. 328-340, 2007.

[18] R. H. Byrd, J. C. Gilbert, and J. Nocedal, "A trust region method based on interior point techniques for nonlinear programming," Mathematical Programming, Series B, vol. 89, no. 1, pp. 149-185, 2000.

[19] A. B. Phillips, M. Haroutunian, S. K. Man, A. J. Murphy, S. W. Boyd, J. I. R. Blake, and G. Griffiths, "Nature in Engineering for Monitoring the Oceans: Comparison of the energetic costs of marine animals and AUVs," in Further Advances in Unmanned Marine Vehicles, G. Roberts and R. Sutton, Eds. IET Control Engineering Series, 2012, pp. 373-405.

\section{ACKNOWLEDGMENT}

The authors would like to thank Stephen Woodward, an expert glider pilot, for his help during the collaborative project. 